\title{
Visible light-mediated difluoroalkylation of electron-deficient alkenes
}

\author{
Vyacheslav I. Supranovich ${ }^{1}$, Vitalij V. Levin ${ }^{1}$, Marina I. Struchkova ${ }^{1}$, Jinbo $\mathrm{Hu}^{2}$ \\ and Alexander D. Dilman*1
}

\section{Letter}

\section{Address:}

${ }^{1}$ N. D. Zelinsky Institute of Organic Chemistry, 119991 Moscow, Leninsky prosp. 47, Russian Federation and ${ }^{2}$ Key Laboratory of Organofluorine Chemistry, Center for Excellence in Molecular Synthesis, Shanghai Institute of Organic Chemistry, University of Chinese Academy of Sciences, Chinese Academy of Sciences, 345 Ling-Ling Road, Shanghai 200032, China

Email:

Alexander D. Dilman* - adil25@mail.ru

* Corresponding author

\section{Keywords:}

difluoroalkylation; organofluorine compounds; radical addition; visible light

\author{
Beilstein J. Org. Chem. 2018, 14, 1637-1641. \\ doi:10.3762/bjoc. 14.139 \\ Received: 11 April 2018 \\ Accepted: 12 June 2018 \\ Published: 02 July 2018 \\ Associate Editor: J. A. Murphy \\ (C) 2018 Supranovich et al.; licensee Beilstein-Institut. \\ License and terms: see end of document.
}

\begin{abstract}
A method for the reductive difluoroalkylation of electron-deficient alkenes using 1,1-difluorinated iodides mediated by irradiation with blue light is described. The reaction involves radical addition of 1,1-difluorinated radicals at the double bond followed by hydrogen atom transfer from sodium cyanoborohydride.
\end{abstract}

\section{Introduction}

Applications of organofluorine compounds in medicinal chemistry and related fields [1,2] have stimulated intensive developments of methods for their synthesis $[3,4]$. Though the major emphasis has long been placed on trifluoromethylated molecules, compounds bearing the difluoromethylene fragment have attracted increasing attention in recent years [5-8]. Thus, besides the bioisosteric relationship with ethereal oxygen [9-11], the $\mathrm{CF}_{2}$-unit can influence the reactivity of adjacent functional groups $[12,13]$ and can affect conformational properties of chain and cyclic molecules $[14,15]$.
Radical fluoroalkylation of double bonds is a well-established approach for the synthesis of organofluorine compounds [1624]. While perfluorinated alkyl iodides and bromides are typically employed, reactions with gem-difluorinated iodides are less elaborated, which is primarily associated with availability issues [25-29]. Recently, we developed a general protocol for the synthesis of iodides $\mathbf{1}$ from organozinc reagents and a source of difluorocarbene [30-32] (Scheme 1). Moreover, it was shown that compounds bearing the $\mathrm{CF}_{2} \mathrm{I}$ group can be obtained from carbonyl compounds and equivalents of the iododifluo- 
romethyl carbanion [33-36]. We also demonstrated that iodides 1 can alkylate silyl enol ethers [37] under photoredox conditions [38-40]. However, the latter protocol is inapplicable to the addition to electron-deficient alkenes since a radical resulting from the addition step cannot be oxidized by photocatalysts. Herein we report a convenient method for performing hydroperfluoroalkylation of electron-deficient alkenes employing iodides 1 mediated by visible light. The reaction proceeds without the use of a photosensitizer or a photocatalyst.

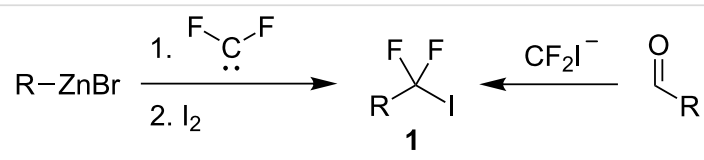

previous work:

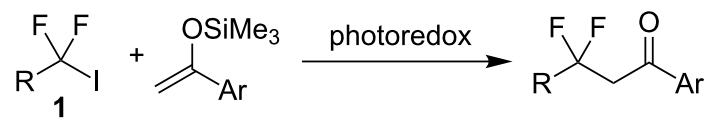

this work:

$$
\underset{\mathrm{R}_{1}}{\mathrm{X}_{\mathrm{EWG}}^{\mathrm{reductant}}} \underset{\text { visible light }}{\stackrel{\text { redug }}{\longrightarrow}}
$$

Scheme 1: Fluoroalkylation of alkenes.

Generation of fluorinated alkyl radicals is typically realized either by single-electron reduction or by radical abstraction of iodine [41-43]. Furthermore, the carbon-iodine bond is prone to homolytic cleavage under UV irradiation [44]. In this regard, we were inspired by the work of Ryu describing the use of energetic UV light (xenon arc lamp) in combination with sodium cyanoborohydride to trigger the reaction of non-fluorinated alkyl iodides [45-47]. Subsequently, we showed that silyldifluo- romethyl iodide can generate the corresponding gem-difluorinated radical in combination with an $\mathrm{N}$-heterocyclic carbene/ borane complex and $400 \mathrm{~nm}$ light [48].

\section{Results and Discussion}

Iodide 1a was selected as a model substrate and its reaction with tert-butyl acrylate (2a) was evaluated in the presence of various boron hydrides (Table 1). The reaction was performed in methanol with irradiation by a strip of blue light emitting diodes. The use of sodium cyanoborohydride provided a moderate yield of product 3a and about $60 \%$ conversion of $\mathbf{1 a}$ (Table 1, entry 1). The addition of pyridine notably increased the yield, presumably, by scavenging boron species with a B-I bond formed in the reaction (Table 1, entry 2). However, some byproducts were observed in amounts of up to $10 \%$, which were tentatively identified as oligomers arising from multiple addition of tert-butyl acrylate to the propagating radical. Fortunately, increase of the amount of borohydride to 2 equiv suppressed the formation of oligomers, and addition product $\mathbf{3 a}$ was isolated in $83 \%$ yield (Table 1 , entry 6 ). $N, N$-Dimethylimidazolidene borane complex $\left(\mathrm{NHC} \cdot \mathrm{BH}_{3}\right)$ was also efficient (Table 1, entry 5), but because of availability and cost issues, sodium cyanoborohydride was used for further studies. We also evaluated the photoredox-mediated hydrofluoroalkylation process using $f a c-\operatorname{Ir}(\mathrm{ppy})_{3}$ as a photocatalyst in combination with a suitable donor of hydrogen atom. With triethylamine, no expected product was observed. At the same time, with Hantzsch ester (3 equiv), which can serve as a single-electron reductant and as a source of hydrogen [16], 54\% of product $3 \mathbf{a}$ was formed. However, difficulties in removing the pyridine byproduct formed from Hantzsch ester and the use of a precious metal photocatalyst make this protocol less practical compared to that with sodium cyanoborohydride.

Table 1: Optimization studies.<smiles>C=CC(=O)O[Ga]Br</smiles>

[B-H], base

blue LED

$\mathrm{MeOH}, \mathrm{rt}, 20 \mathrm{~h}$<smiles>O=C(CCC(F)(F)Cc1ccccc1)O[Ga]</smiles>

\begin{tabular}{llll}
\hline entry & hydride (equiv) & base (equiv) & yield, \%a \\
\hline 1 & $\mathrm{NaBH}_{3} \mathrm{CN}(1.5)$ & - & 46 \\
2 & $\mathrm{NaBH}_{3} \mathrm{CN}(1.5)$ & pyridine (1.5) & $77^{\mathrm{b}}$ \\
3 & $\mathrm{Py}^{\mathrm{B} \mathrm{BH}_{3}(1.5)}$ & pyridine (1.5) & $78^{\mathrm{b}}$ \\
4 & $\mathrm{Py}^{\mathrm{B} H}(2.0)$ & pyridine (2.0) & $80^{\mathrm{b}}$ \\
5 & $\mathrm{NHC}_{3} \mathrm{BH}_{3}(1.5)$ & pyridine (1.5) & $76^{\mathrm{c}}$ \\
6 & $\mathrm{NaBH}_{3} \mathrm{CN}(2.0)$ & pyridine (1.5) & $83^{\mathrm{c}}$ \\
7 & $\mathrm{NaBH}_{3} \mathrm{CN}(2.0)$ & NaOAc (1.5) & $74^{\mathrm{b}}$ \\
\hline
\end{tabular}

${ }^{a}$ Determined by ${ }^{19} \mathrm{~F}$ NMR with internal standard. ${ }^{b}$ Oligomeric byproducts $(5-11 \%)$ were observed. ${ }^{\mathrm{C}}$ Isolated yield. 
Under the optimized conditions a series of gem-difluorinated iodides 1 were coupled with alkenes 2 (Figure 1). Esters and amides of acrylic acid, acrylonitrile, and vinyl phosphonate used in a small excess (1.2 equiv) were successfully fluoroalkylated. For vinyl(phenyl)sulfone, significant amounts of hydrodeiodination product $\mathrm{R}_{\mathrm{f}} \mathrm{H}$ was formed, likely because of the decreased reactivity of the sulfone double bond toward fluorinated radicals. In this case, two equivalents of the alkene have to be used to achieve good yields of products $3 \mathbf{t}, \mathbf{u}$. It should also be pointed out that the reaction tolerates aromatic bromide substituents (products $\mathbf{3 b}, \mathbf{c}, \mathbf{n}, \mathbf{o}$ ), a boryl fragment (product 3d) and unprotected hydroxy groups (products $\mathbf{3 i}, \mathbf{j}, \mathbf{m}$ ). Electronrich and neutral alkenes were ineffective in this reaction. Indeed, 1-phenyl-(1-trimethylsilyloxy)ethylene and 4-phenylbut-1-ene gave no hydrofluoroalkylation products when combined with iodide 1a under standard conditions.

The proposed mechanism is shown in Scheme 2. First, difluorinated iodide $\mathbf{1}$ interacts with boron hydride to form a small equilibrium concentration of a halogen-bonded complex $[49,50]$. This complex is activated by light to effect homolytic

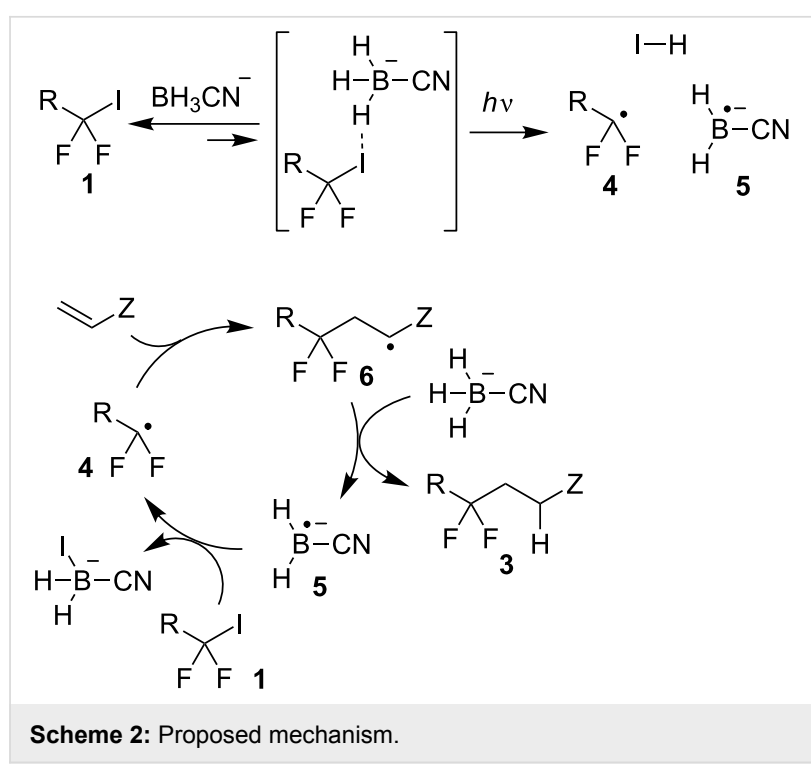

cleavage of the carbon-iodine bond with the formation of radical 4 and boron-centered radical anion $\mathbf{5}$. After the initiation event, the reaction proceeds via a chain mechanism. Thus,

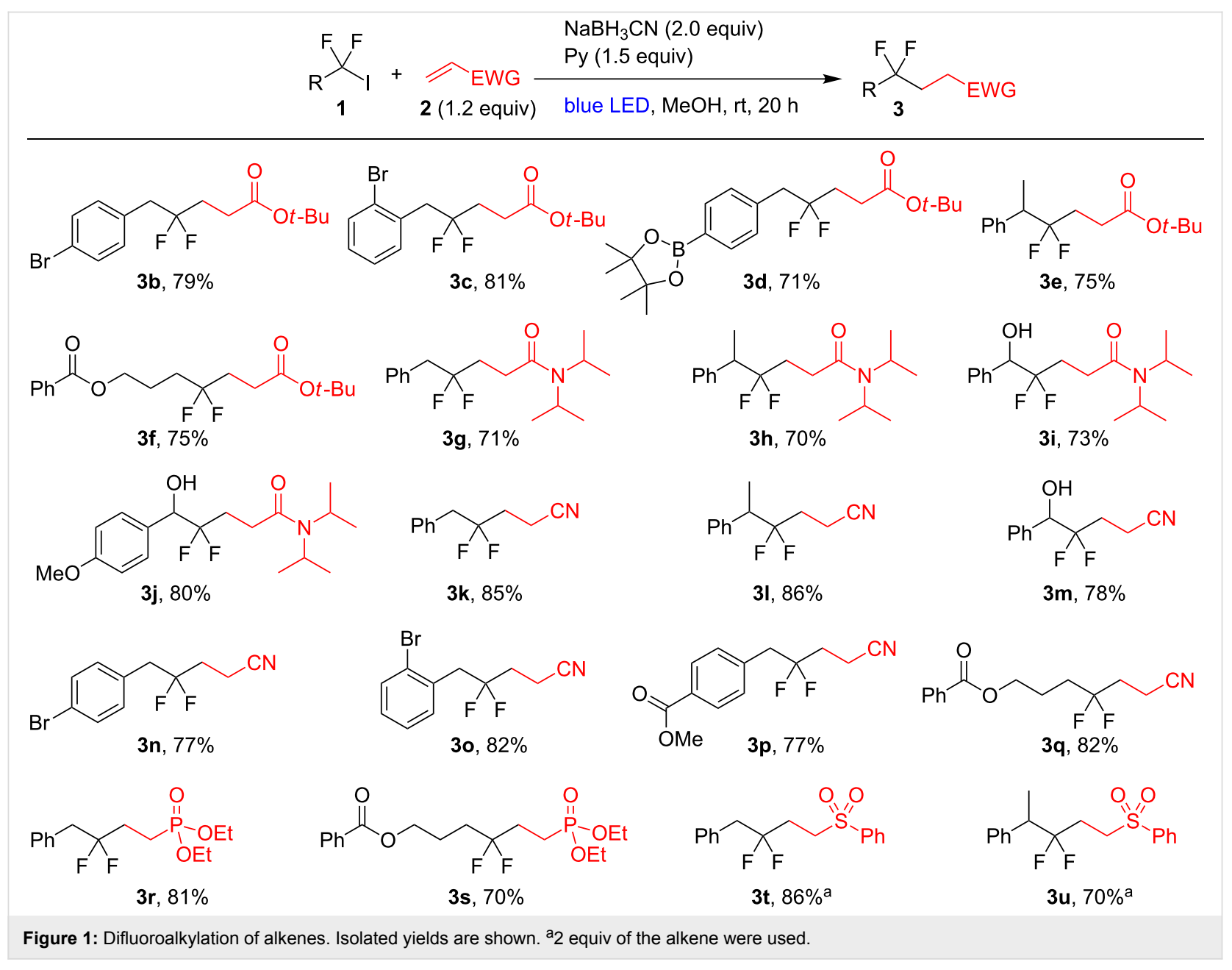


radical addition at the double bond gives radical $\mathbf{6}$, which abstracts a hydrogen atom from cyanoborohydride to generate boryl radical anion $\mathbf{5}$. The latter species can readily abstract the iodine atom from starting iodide $\mathbf{1}$ which results in the formation of radical 4 [51,52].

\section{Conclusion}

In summary, a convenient method for the synthesis of gemdifluorinated compounds via hydrofluoroalkylation of electrondeficient alkenes is described. Readily available boron cyanohydride is used as a source of hydrogen and as a trigger for the generation of free radicals mediated by visible light.

\section{Supporting Information}

\section{Supporting Information File 1}

Full experimental details, compound characterization, and copies of NMR spectra.

[https://www.beilstein-journals.org/bjoc/content/ supplementary/1860-5397-14-139-S1.pdf]

\section{Acknowledgements}

This work was supported by the Russian Foundation for Basic Research (project 18-53-53009).

\section{ORCID ${ }^{\circledR}$ iDs}

Alexander D. Dilman - https://orcid.org/0000-0001-8048-7223

\section{References}

1. Wang, J.; Sánchez-Roselló, M.; Aceña, J. L.; del Pozo, C.; Sorochinsky, A. E.; Fustero, S.; Soloshonok, V. A.; Liu, H. Chem. Rev. 2014, 114, 2432-2506. doi:10.1021/cr4002879

2. Purser, S.; Moore, P. R.; Swallow, S.; Gouverneur, V. Chem. Soc. Rev. 2008, 37, 320-330. doi:10.1039/B610213C

3. Liang, T.; Neumann, C. N.; Ritter, T. Angew. Chem., Int. Ed. 2013, 52, 8214-8264. doi:10.1002/anie.201206566

4. Groult, H.; Leroux, F. R.; Tressaud, A., Eds. Modern Synthesis Processes and Reactivity of Fluorinated Compounds; Elsevier: Amsterdam, 2017.

5. Belhomme, M.-C.; Besset, T.; Poisson, T.; Pannecoucke, X. Chem. - Eur. J. 2015, 21, 12836-12865. doi:10.1002/chem.201501475

6. Krishnamoorthy, S.; Prakash, G. K. S. Synthesis 2017, 49, 3394-3406. doi:10.1055/s-0036-1588489

7. Dilman, A. D.; Levin, V. V. Mendeleev Commun. 2015, 25, 239-244. doi:10.1016/j.mencom.2015.07.001

8. Gao, B.; Ni, C.; Hu, J. Chimia 2014, 68, 414-418. doi:10.2533/chimia.2014.414

9. Blackburn, G. M.; England, D. A.; Kolkmann, F. J. Chem. Soc., Chem. Commun. 1981, 930-932. doi:10.1039/c39810000930

10. Xu, Y.; Aoki, J.; Shimizu, K.; Umezu-Goto, M.; Hama, K.; Takanezawa, Y.; Yu, S.; Mills, G. B.; Arai, H.; Qian, L.; Prestwich, G. D. J. Med. Chem. 2005, 48, 3319-3327. doi:10.1021/jm049186t
11. Ivanova, M. V.; Bayle, A.; Besset, T.; Pannecoucke, X.; Poisson, T. Chem. - Eur. J. 2016, 22, 10284-10293. doi:10.1002/chem.201601310

12. Baskin, J. M.; Prescher, J. A.; Laughlin, S. T.; Agard, N. J.; Chang, P. V.; Miller, I. A.; Lo, A.; Codelli, J. A.; Bertozzi, C. R. Proc. Natl. Acad. Sci. U. S. A. 2007, 104, 16793-16797. doi:10.1073/pnas.0707090104

13. Codelli, J. A.; Baskin, J. M.; Agard, N. J.; Bertozzi, C. R. J. Am. Chem. Soc. 2008, 130, 11486-11493. doi:10.1021/ja803086r

14. O'Hagan, D.; Wang, Y.; Skibinski, M.; Slawin, A. M. Z. Pure Appl. Chem. 2012, 84, 1587-1595. doi:10.1351/PAC-CON-11-09-26

15. Wang, Y.; Callejo, R.; Slawin, A. M. Z.; O'Hagan, D. Beilstein J. Org. Chem. 2014, 10, 18-25. doi:10.3762/bjoc.10.4

16. Sumino, S.; Uno, M.; Fukuyama, T.; Ryu, I.; Matsuura, M.; Yamamoto, A.; Kishikawa, Y. J. Org. Chem. 2017, 82, 5469-5474. doi:10.1021/acs.joc.7b00609

17. Zhu, L.; Wang, L.-S.; Li, B.; Fu, B.; Zhang, C.-P.; Li, W. Chem. Commun. 2016, 52, 6371-6374. doi:10.1039/C6CC01944G

18. Zhou, S.-H.; Lin, J.-H.; Zhao, G.; Xiao, J.-C.; Cao, W.-G. RSC Adv. 2016, 6, 60080-60083. doi:10.1039/C6RA11557H

19. Lin, Q.-Y.; Xu, X.-H.; Qing, F.-L. Org. Biomol. Chem. 2015, 13, 8740-8749. doi:10.1039/C5OB01302J

20. Mizuta, S.; Verhoog, S.; Engle, K. M.; Khotavivattana, T.; O'Duill, M.; Wheelhouse, K.; Rassias, G.; Médebielle, M.; Gouverneur, V. J. Am. Chem. Soc. 2013, 135, 2505-2508. doi:10.1021/ja401022x

21. Wu, X.; Chu, L.; Qing, F.-L. Angew. Chem., Int. Ed. 2013, 52, 2198-2202. doi:10.1002/anie.201208971

22. Wilger, D. J.; Gesmundo, N. J.; Nicewicz, D. A. Chem. Sci. 2013, 4 , 3160-3165. doi:10.1039/c3sc51209f

23. Erdbrink, H.; Peuser, I.; Gerling, U. I. M.; Lentz, D.; Koksch, B.; Czekelius, C. Org. Biomol. Chem. 2012, 10, 8583-8586. doi:10.1039/c2ob26810h

24. Beier, P.; Voltrová, S. Fluorine Notes 2017, 115, 3-4. doi:10.17677/fn20714807.2017.06.02

25. Yang, Z.-Y. J. Org. Chem. 2004, 69, 2394-2403. doi:10.1021/jo0354488

26. Li, A.-R.; Chen, Q.-Y. Synthesis 1997, 1481-1488. doi:10.1055/s-1997-1378

27. Li, A.-R.; Chen, Q.-Y. J. Fluorine Chem. 1997, 82, 151-155. doi:10.1016/S0022-1139(96)03545-2

28. Li, A.-R.; Chen, Q.-Y. J. Fluorine Chem. 1997, 81, 99-101. doi:10.1016/S0022-1139(96)03510-5

29. Mikami, K.; Tomita, Y.; Itoh, Y. Angew. Chem., Int. Ed. 2010, 49, 3819-3822. doi:10.1002/anie.201000435

30. Levin, V. V.; Zemtsov, A. A.; Struchkova, M. I.; Dilman, A. D. Org. Lett. 2013, 15, 917-919. doi:10.1021/ol400122k

31. Levin, V. V.; Zemtsov, A. A.; Struchkova, M. I.; Dilman, A. D. J. Fluorine Chem. 2015, 171, 97-101. doi:10.1016/j.jluchem.2014.08.021

32. Dilman, A. D.; Levin, V. V. Acc. Chem. Res. 2018, 51, 1272-1280. doi:10.1021/acs.accounts.8b00079

33. Zhao, Y.; Gao, B.; Hu, J. J. Am. Chem. Soc. 2012, 134, 5790-5793. doi:10.1021/ja301601b

34. Miao, W.; Ni, C.; Zhao, Y.; Hu, J. Org. Lett. 2016, 18, 2766-2769. doi:10.1021/acs.orglett.6b01258

35. Kosobokov, M. D.; Levin, V. V.; Struchkova, M. I.; Dilman, A. D. Org. Lett. 2014, 16, 3784-3787. doi:10.1021/ol501674n

36. Levin, V. V.; Smirnov, V. O.; Struchkova, M. I.; Dilman, A. D. J. Org. Chem. 2015, 80, 9349-9353. doi:10.1021/acs.joc.5b01590 
37. Chernov, G. N.; Levin, V. V.; Kokorekin, V. A.; Struchkova, M. I.; Dilman, A. D. Adv. Synth. Catal. 2017, 359, 3063-3067. doi:10.1002/adsc.201700423

38. Panferova, L. I.; Tsymbal, A. V.; Levin, V. V.; Struchkova, M. I.; Dilman, A. D. Org. Lett. 2016, 18, 996-999.

doi:10.1021/acs.orglett.6b00117

39. Scherbinina, S. I.; Fedorov, O. V.; Levin, V. V.; Kokorekin, V. A.; Struchkova, M. I.; Dilman, A. D. J. Org. Chem. 2017, 82, 12967-12974. doi:10.1021/acs.joc.7b02467

40. Panferova, L. I.; Smirnov, V. O.; Levin, V. V.; Kokorekin, V. A.; Struchkova, M. I.; Dilman, A. D. J. Org. Chem. 2017, 82, 745-753. doi:10.1021/acs.joc.6b02344

41. Chatterjee, T.; Iqbal, N.; You, Y.; Cho, E. J. Acc. Chem. Res. 2016, 49, 2284-2294. doi:10.1021/acs.accounts.6b00248

42. Koike, T.; Akita, M. Top. Catal. 2014, 57, 967-974. doi:10.1007/s11244-014-0259-7

43. Baguley, P. A.; Walton, J. C. Angew. Chem., Int. Ed. 1998, 37, 3072-3082. doi:10.1002/(SICI)1521-3773(19981204)37:22<3072::AID-ANIE3072>3 .0.CO;2-9

44. Uma, S.; Das, P. K. Can. J. Chem. 1994, 72, 865-869. doi:10.1139/v94-113

45. Ryu, I.; Uehara, S.; Hirao, H.; Fukuyama, T. Org. Lett. 2008, 10 , 1005-1008. doi:10.1021/ol7031043

46. Kawamoto, T.; Uehara, S.; Hirao, H.; Fukuyama, T.; Matsubara, H.; Ryu, I. J. Org. Chem. 2014, 79, 3999-4007. doi:10.1021/j0500464q

47. Kawamoto, T.; Ryu, I. Org. Biomol. Chem. 2014, 12, 9733-9742. doi:10.1039/C4OB01784F

48. Supranovich, V. I.; Levin, V. V.; Struchkova, M. I.; Korlyukov, A. A.; Dilman, A. D. Org. Lett. 2017, 19, 3215-3218. doi:10.1021/acs.orglett.7b01334

49. Cavallo, G.; Metrangolo, P.; Milani, R.; Pilati, T.; Priimagi, A.; Resnati, G.; Terraneo, G. Chem. Rev. 2016, 116, 2478-2601. doi:10.1021/acs.chemrev.5b00484

50. Beale, T. M.; Chudzinski, M. G.; Sarwar, M. G.; Taylor, M. S. Chem. Soc. Rev. 2013, 42, 1667-1680. doi:10.1039/C2CS35213C

51. Kawamoto, T.; Fukuyama, T.; Ryu, I. J. Am. Chem. Soc. 2012, 134, 875-877. doi:10.1021/ja210585n

52. Sheeller, B.; Ingold, K. U. J. Chem. Soc., Perkin Trans. 2 2001, 480-486. doi:10.1039/b009494n

\section{License and Terms}

This is an Open Access article under the terms of the Creative Commons Attribution License (http://creativecommons.org/licenses/by/4.0), which permits unrestricted use, distribution, and reproduction in any medium, provided the original work is properly cited.

The license is subject to the Beilstein Journal of Organic Chemistry terms and conditions: (https://www.beilstein-journals.org/bjoc)

The definitive version of this article is the electronic one which can be found at:

doi:10.3762/bjoc. 14.139 